\title{
Embryonal rhabdomyosarcoma of liver in a 16-year-old boy: A rare case report
}

\author{
Rajamahendran Rajendran, Amudhan A., Prabhakaran R., \\ Kannan D., Chandramohan S.M.
}

\begin{abstract}
Introduction: Primitive mesenchymal tumors represent about 9-15\% of all hepatic tumors in children. Only about 150 cases have been reported in literature so far. Herein, we report a case of ruptured embryonal rhabdomyosarcoma. Case Report: A 16-year-old boy, with weight of $26 \mathrm{~kg}$, presented to us with sudden onset of abdominal pain and fever. Clinical examination revealed a tender hepatomegaly about $6 \mathrm{~cm}$ below right costal margin. Contrast-enhanced computed tomography scan of abdomen revealed 15x11x9 cm heterogenous multi-septated loculated mass lesion involving right lobe of liver involving segments 5, 6, 7, 8. Diagnosticlaparoscopy showed a tumor occupying the entire right lobe of liver with no other deposits. The tumor capsule was
\end{abstract}

Rajamahendran Rajendran ${ }^{1}$, Amudhan A. ${ }^{2}$, Prabhakaran R. ${ }^{2}$, Kannan D. ${ }^{3}$, Chandramohan S.M. ${ }^{4}$

Affiliations: ${ }^{1}$ Post Graduate in Surgical Gastroenterology, Institute of Surgical Gastroenterology, Rajiv Gandhi Government General Hospital, Chennai, Tamil Nadu, India; ${ }^{2}$ Assistant Professor in Surgical Gastroenterology, Institute of Surgical Gastroenterology, Rajiv Gandhi Government General Hospital, Chennai, Tamil Nadu, India; ${ }^{3}$ Professor of Surgical Gastroenterology, Institute of Surgical Gastroenterology, Rajiv Gandhi Government General Hospital, Chennai, Tamil Nadu, India; ${ }^{4} \mathrm{Head}$ of Department of Surgcial Gastroenterology, Institute of Surgical Gastroenterology, Rajiv Gandhi Government General Hospital, Chennai, Tamil Nadu, India.

Corresponding Author: Professor D. Kannan, No. 245, Tower 2, 4th Floor, Rajiv Gandhi Government General Hospital, Chennai-600003, Tamil Nadu, India; Ph: 919787387183; Email: minnalraja@gmail.com

Received: 11 August 2014

Accepted: 03 September 2014

Published: 28 October 2014 seen ruptured on the right lateral side for which we did emergency right hepatectomy. Weight of the specimen was $2.8 \mathrm{~kg}$. Histopathology revealed the lesion as embryonal rhabdomyosarcoma with co-existing mesenchymal hamartoma. Immunohistochemistry showed Vimentin and Desmin positive. Patient was on carboplatin and Ifosfamide chemotherapy. Patient expired on 3oth postoperative day due to acute respiratory distress. Conclusion: Improved survival can be expected only if we detect the disease early. This case report will enlighten this type of rare tumor in young children and an early referral to the specialist.

Keywords: Primary embryonal rhabdomyosarcoma, Heterogenous mass in liver, Ruptured capsule, Right Hepatectomy, Liver

\section{How to cite this article}

R Rajamahendran, Amudhan A, Prabhakaran R, Kannan D, Chandramohan SM. Embryonal rhabdomyosarcoma of liver in a 16-year-old boy: A rare case report. Int $\mathrm{J}$ Hepatobiliary Pancreat Dis 2014;4:52-56.

Article ID: 100022IJHPDRR2014

$* * * * * * * * *$

doi:10.5348/ijhpd-2014-22-CR-10

\section{INTRODUCTION}

Primary liver tumors rank the third amongst the solid malignant tumors in children following Wilms tumor and Neuroblastoma. They account for $2 \%$ of total solid malignancies in pediatric cases [1]. Primitive 
mesenchymal tumors rank the fourth among the malignant tumors of pediatric age group following hepatoblastoma, infantile hemangioendothelioma and hepatocellular carcinoma. Rhabdomyosarcoma is the malignant solid tumor arising from mesenchymal tissues which normally differentiates to form striated muscle. It is most common tumor in the age group less than 15 years. There are four types of rhabdomyosarcomapleomorphic, embryonal, alveolar and botryoidal. The 5 -year survival rate of localized disease is $80 \%$ and for metastatic diseases $5 \%$

Primitive mesenchymal tumors represent about 9-15\% of all hepatic tumors in children. So far only 150 cases of rhabdomyosarcoma liver have been reported in literature. Herein, we report a case of ruptured primary embryonal rhabdomyosarcoma of liver in a 16-year-old boy. Embryonal type of rhabdomyosarcoma of liver is very rare and only 12 cases were reported in literature so far.

\section{CASE REPORT}

A 16-year-old boy with body weight of $26 \mathrm{~kg}$ was presented to us with a mass in the right hypochondrium since one month with history of abdominal pain, fever with chills and rigors. He had no history of jaundice, weight loss or loss of appetite. On elaborating the history, the mass was present for the last one month and rapidly increased for the last one week with severe intolerable pain last four days. On clinical examination, abdomen was soft and minimal guarding was present. A firm tender hepatomegaly was present $6 \mathrm{~cm}$ below right costal margin. Surface found to be smooth and edges were rounded. Contrast-enhanced computed tomography scan of abdomen revealed 15x11x9 cm heterogeneous multi-septated lobulated mass lesion involving right lobe of liver involving the segments 5, 6, 7, 8 (Figures 1 and 2). Portal venous Doppler study showed displaced and stretched right portal vein with normal flow. Laboratory investigations at the time of admission showed hemoglobin $8 \mathrm{~g} / \mathrm{dL}$, platelets 4 lakhs $/ \mathrm{mm}^{3}$, blood sugar $80 \mathrm{mg} / \mathrm{dL}$, blood urea $22 \mathrm{mg} / \mathrm{dL}$ and serum creatinine

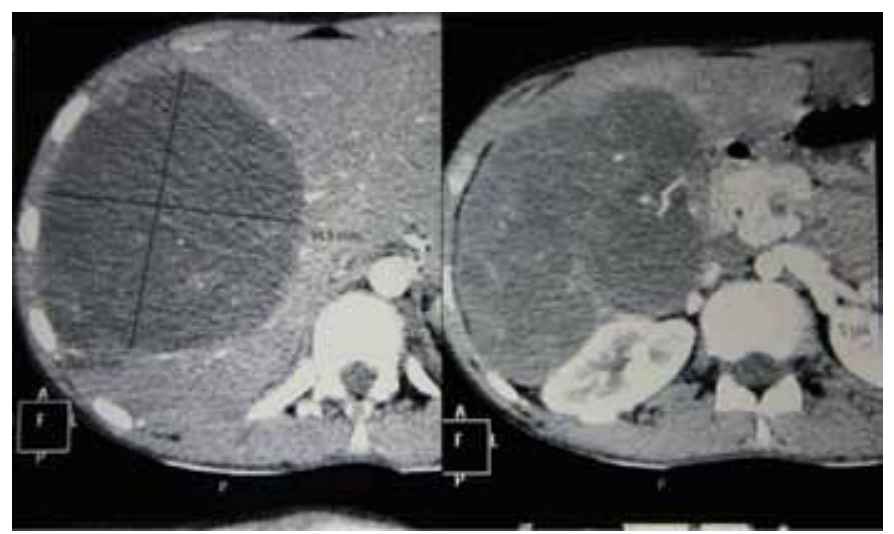

Figure 1: Contrast-enhanced computed tomography scan of abdomen showing heterogenous mass lesion in right lobe of liver.
$0.8 \mathrm{mg} / \mathrm{dL}$. Two units of packed cells were transfused and the preoperative hemoglobin was $10 \mathrm{mg} / \mathrm{dl}$. Tumor markers AFP and CA 19-9 were done. Alpha fetoprotein value was $4 \mathrm{ng} / \mathrm{mL}$ and CA 19-9 was found to $20 \mathrm{U} / \mathrm{mL}$. Liver biopsy was not taken to avoid the risk of seedling of an operable tumor.

Diagnostic laparoscopy revealed a tumor occupying the segments $5,6,7,8$ with ruptured capsule in the right lateral lobe of liver (Figure 3 ). There was no other

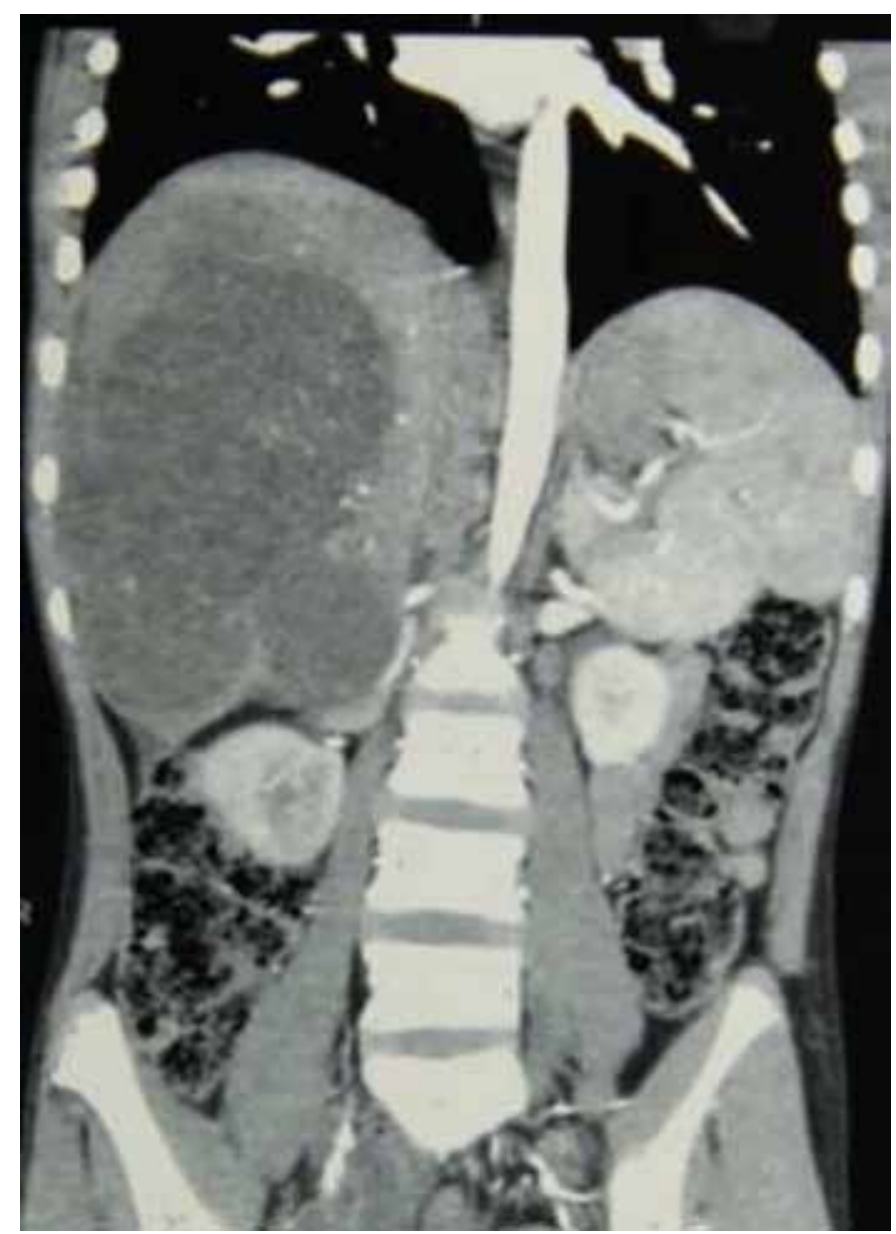

Figure 2: Coronal view of contrast-enhanced computed tomography scan of abdomen showing the lesion occupying the entire right lobe.

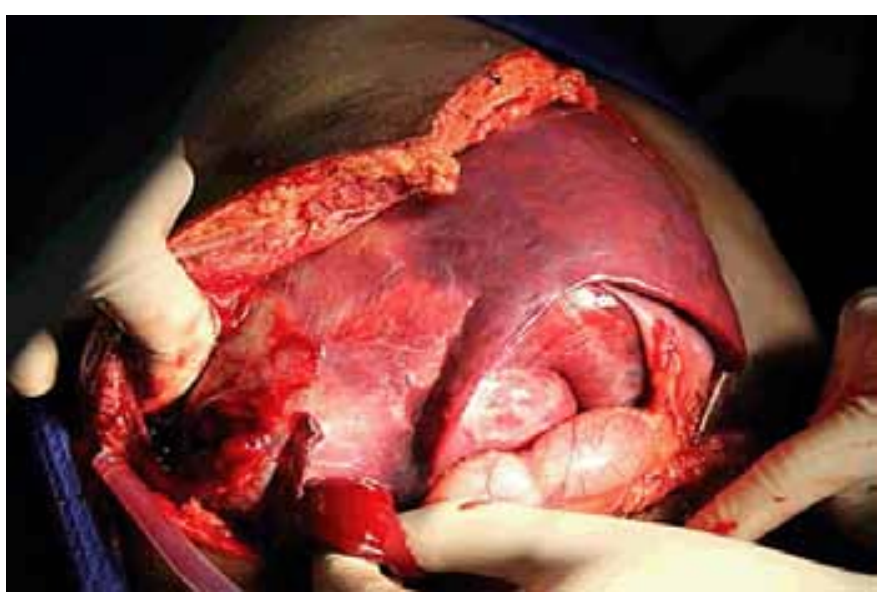

Figure 3: Intraoperative picture showing ruptured capsule of tumor. 
peritoneal or omental mets. The left lobe of liver was found normal with other lesions. So we decided to proceed with right hepatectomy. The inflow control was done with Pringles manoeuvre. Parenchymal dissection was done with Harmonic scalpel and Kelly crush clamp technique (Figures 4 and 5). The bleeding vessels from raw area in the left lobe were ligated with 3'o Silk sutures. Specimen retrieved and complete hemostasis was achieved in the remnant liver raw area. Weight of the specimen was $2.8 \mathrm{~kg}$ (Figure 6).

Histopathology showed liver parenchyma with tumor composed of round to polyhedral and spindle shaped cells with bizarre nuclei, arranged in sheets and clusters with vague lobular pattern in some places. Tumor cells inhibit intracytoplasmic globules and multinucleate giant cells with atypical mitosis. Entrapped bile ductules are seen. Stroma showed extensive hemorrhagic areas with areas of necrosis. The above features are suggestive of embryonal variety of rhabdomyosarcoma probably coexisting with mesenchymal hamartoma (Figure $7 \mathrm{~A}-\mathrm{B}$ ), resected margins were free of tumor.

IHC markers are positive for Vimentin showed focal positivity, Desmin showed diffuse strong positivity, CK 7 glands showed positivity. CD 34 was found negative. All

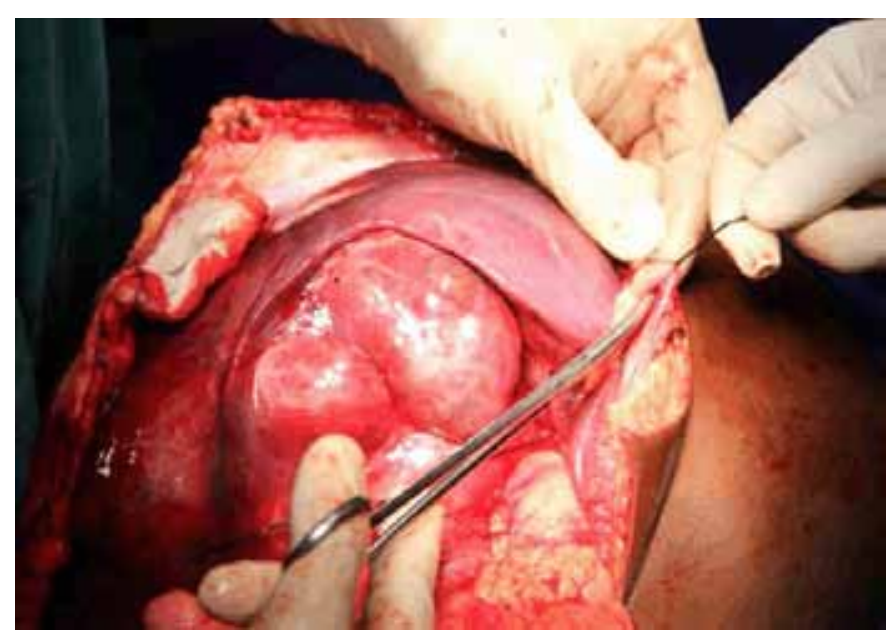

Figure 4: Rhabdomyosarcoma, during surgery, tying the Falciparum ligament.

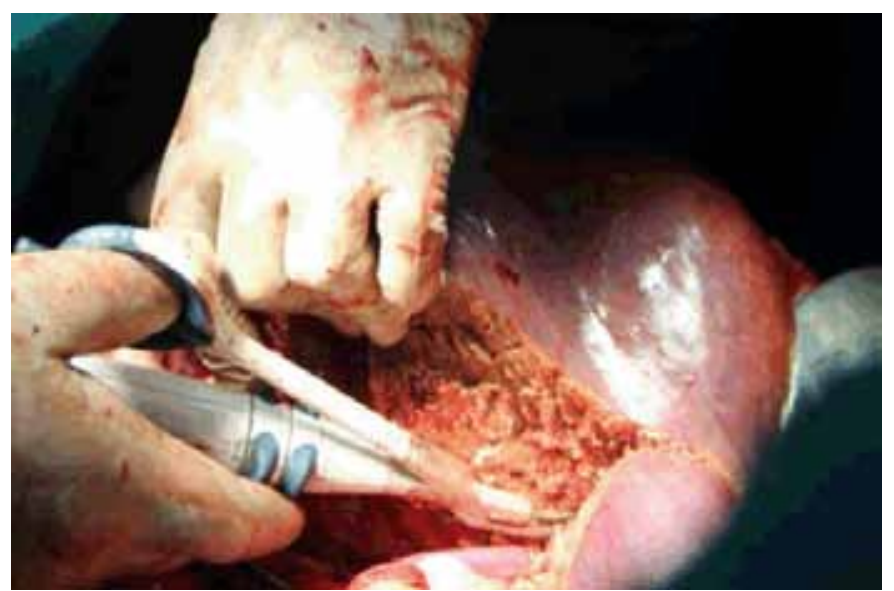

Figure 5: Parenchymal resection using Harmonic scalpel.

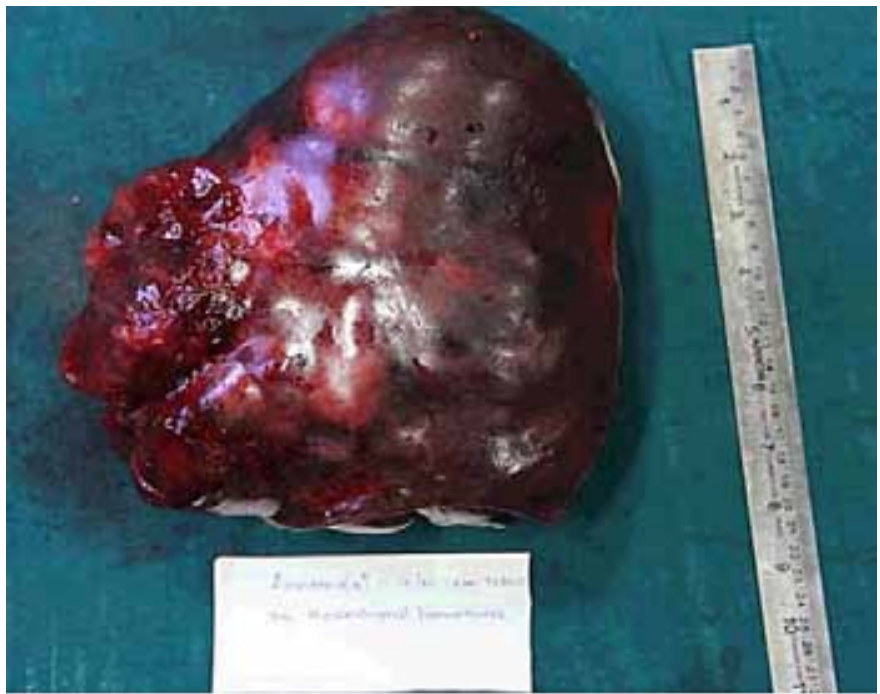

Figure 6: Postoperative specimen of rhabdomyosarcoma liver.
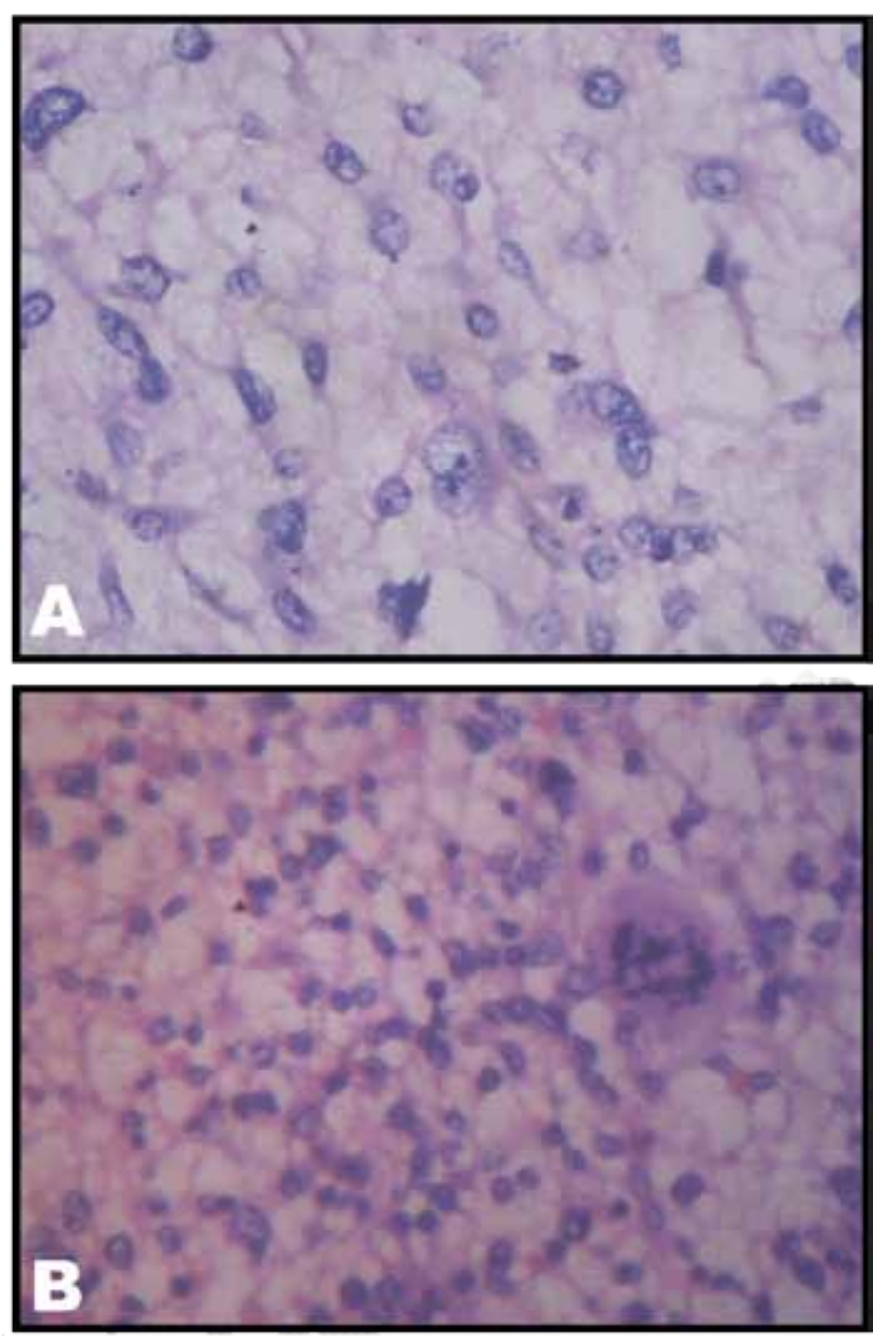

Figure 7: Histopathology examination showing (A) Pleomorphic nuclei and spindle cells, (B) Liver parenchyma with mitotic figures. 
these confirmed the diagnosis of embryonal variety of rhabdomyosarcoma. First cycle of chemotherapy started with ifosfamide at $800 \mathrm{mg} /$ day and carboplatin at 300 $\mathrm{mg} /$ day.

Patient expired on 3oth postoperative day suddenly because of respiratory distress and pleural effusion. Postmortem examination was not done.

\section{DISCUSSION}

Having an incidence of $9-15 \%$ in children, hepatic rhabdomyosarcoma liver needs a lot of experience to diagnose. Rhabdomyosarcoma was classified into four types-pleomorphic, alveolar, embryonal and botryoidal types. The predominant age of presentation are pleomorphic type in male adults, alveolar type in adolescents and young adults, embryonal type in infants and children and botryoidal type in young children. The different types of rhabdomyosarcoma can be differentiated on the basis of histopathological appearance. The tumor cells are more elongated and less densely cellular in embryonal type. In alveolar type the tumor cells tend to be smaller and rounder, often with a denser cellularity, and are so named because of their resemblance to the appearance of the small air sacs in the lungs (the "alveoli"). Rhabdomyosarcoma have very poor prognosis with a 5 -year survival rate of $80 \%$ for localized disease and $5 \%$ for metastatic disease.

These cases have a great challenge in treatment because of the rarity of the disease and absence of clinical trials and standard treatment guidelines. A lot of case reports published showed that most patients expired within 5 years of the diagnosis. Neoadjuvant chemotherapy was found to extend the survival of these patients [2]. Postoperative chemotherapy is advised for all patients. Various chemotherapy regimens are applied for these patients like vinorelbine and cyclophosphamide, gemcitabine and docetaxel, rapamycin, topotecan and vincristine [3, 4]. Our patient received ifosfamide and carboplatin for 1 cycle, but unfortunately patient suddenly died of acute respiratory distress and pleural effusion.

With this case report, we would like to stress the aggressive nature of this disease and the necessity for early detection of the disease. A multidisciplinary approach is needed in managing patients as there is no protocol available. An extensive workup is essential because these tumors have the potential to grow rapidly. PET CT scan may be of beneficial in these patients before surgery [5] and may help in diagnosing the metastasis before taking up for hepatectomy.

\section{CONCLUSION}

The poor prognosis and early death of most cases reported so far implies the necessity of early detection and evaluation of the disease. Surgical resection along with chemotherapy provides improved survival only if given in the early stage of the disease.

$$
* * * * * * * * *
$$

\section{Author Contributions}

Rajamahendran Rajendran - Substantial contributions to conception and design, Acquisition of data, Analysis and interpretation of data, Drafting the article, Revising it critically for important intellectual content, Final approval of the version to be published

Amudhan A. - Analysis and interpretation of data, Revising it critically for important intellectual content, Final approval of the version to be published

Prabhakaran R. - Substantial contributions to conception and design, Revising it critically for important intellectual content, Final approval of the version to be published Kannan D. - Substantial contributions to conception and design, Revising it critically for important intellectual content, Final approval of the version to be published Chandramohan S.M. - Substantial contributions to conception and design, Revising it critically for important intellectual content, Final approval of the version to be published

\section{Guarantor}

The corresponding author is the guarantor of submission.

\section{Conflict of Interest}

Authors declare no conflict of interest.

\section{Copyright}

(C) 2014 Rajamahendran Rajendran et al. This article is distributed under the terms of Creative Commons Attribution License which permits unrestricted use, distribution and reproduction in any medium provided the original author(s) and original publisher are properly credited. Please see the copyright policy on the journal website for more information.

\section{REFERENCES}

1. Schoofs G, Braeye L, Vanheste R, Verswijvel G, DebiecRychter M, Sciot R. Hepatic rhabdomyosarcoma in an adult: A rare primary malignant liver tumor. Case report and literature review. Acta Gastroenterol Belg 2011 Dec;74(4):576-81.

2. Almogy G, Lieberman S, Gips $M$, et al. Clinical outcomes of surgical resections for primary liver sarcoma in adults: Results from a single centre. Eur $\mathrm{J}$ Surg Oncol 2004 May;30(4):421-7.

3. Geel JA, Loveland JA, Pitcher GJ, Beale P, Kotzen J, Poole JE. Management of undifferentiated embryonal sarcoma of the liver in children: A case series and management review. S Afr Med J 2013 Jun 27;103(10):728-31.

4. Undifferentiated Embryonal sarcoma of liverCombination treatment by surgery and chemotherapy. Dae-Yeon kim, Ki- Hong Kim, Seoul Korea, presented 
in 34th annual meeting of pacific associations of paediatric surgeons, Japan.
5. Haider N, Nadim MS, Piracha MN. Primary embryonal rhabdomyosarcoma of the liver in a young male. $J$ Coll Physicians Surg Pak 2013 Oct;23(10):750-1.
Access full text article on other devices

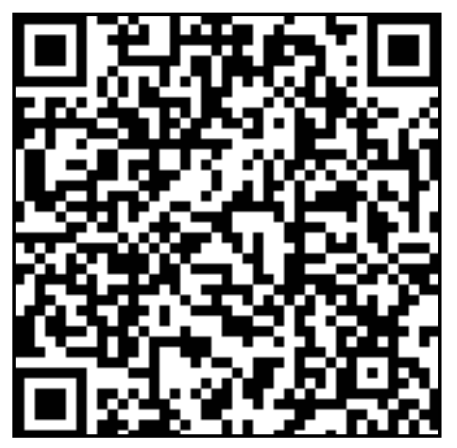

Access PDF of article on other devices

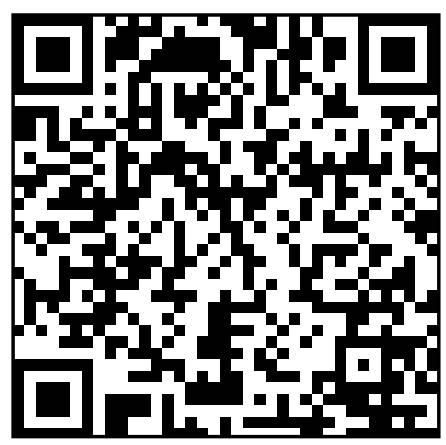

